Research Paper

\title{
Steroid-induced osteonecrosis of the femoral head reveals enhanced reactive oxygen species and hyperactive osteoclasts
}

\author{
Kai Chen ${ }^{*}$, Yuhao Liu1, $1,3^{*}$, Jianbo He1,3, Nathan Pavlos ${ }^{1}$, Chao Wang11, Jacob Kenny', Jinbo Yuan ${ }^{1}$, Qingwen \\ Zhang ${ }^{2,3}$, Jiake $\mathrm{Xu}^{1,2 \llbracket}$, Wei He ${ }^{2,3,4}$ \\ 1. School of Biomedical Sciences, The University of Western Australia, Perth, WA 6009, Australia \\ 2. Department of Joint Orthopaedics, The First Affiliated Hospital, Guangzhou University of Chinese Medicine, Guangzhou, Guangdong 510405, China \\ 3. The Lab of Orthopaedics of Chinese Medicine, Lingnan Medical Research Center, Guangzhou University of Chinese Medicine, Guangzhou, Guangdong \\ 510405, China \\ 4. Research Institute of Orthopaedics of Chinese Medicine, Guangzhou University of Chinese Medicine, Guangzhou, Guangdong 510378, China \\ *Kai Chen and Yuhao Liu contributed equally to this work. \\ $\bowtie$ Corresponding authors: Prof. Wei He. Email: hw13802516062@163.com; Prof. Jiake Xu. Email: jiake.xu@uwa.edu.au
}

(1) The author(s). This is an open access article distributed under the terms of the Creative Commons Attribution License (https://creativecommons.org/licenses/by/4.0/). See http://ivyspring.com/terms for full terms and conditions.

Received: 2019.10.05; Accepted: 2020.03.18; Published: 2020.04.06

\begin{abstract}
Steroid-induced osteonecrosis of the femoral head (ONFH) is a progressive bone disorder which typically results in femoral head collapse and hip joint dysfunction. It is well-accepted that abnormal osteoclast activity contributes to loss of bone structural integrity and subchondral fracture in ONFH. However, the pathophysiologic mechanisms underlying the recruitment and hyperactivation of osteoclasts in ONFH remain incompletely understood. We assessed the changes of reactive oxygen species (ROS) level and subsequent osteoclast alterations in steroid-induced osteonecrotic femoral heads from both patients and rat ONFH models. When compared with healthy neighboring bone, the necrotic region of human femoral head was characterized by robust up-regulated expression of osteoclast-related proteins [cathepsin $\mathrm{K}$ and tartrate-resistant acid phosphatase(TRAP)] but pronounced down-regulation of antioxidant enzymes (catalase, $Y$-glutamylcysteine synthetase [Y-GCSc], and superoxide dismutase 1 [SODI]). In addition, the ratio of TNFSFII (encoding RANKL)/TNFRSFI IB (encoding OPG) was increased within the necrotic bone. Consistently, in rat ONFH models induced by methylprednisolone (MPSL) and imiquimod (IMI), significant bone loss in the femoral head was observed, attributable to increased numbers of TRAP positive osteoclasts. Furthermore, the decreased expression of antioxidant enzymes observed by immunoblotting was accompanied by increased ex-vivo ROS fluorescence signals of dihydroethidium (DHE) in rat ONFH models. Therefore, this study lends support to the rationale that antioxidant agents may be a promising therapeutic avenue to prevent or mitigate the progression of steroid-induced ONFH by inhibiting ROS level and hyperactive osteoclasts.
\end{abstract}

Key words: Reactive oxygen species (ROS); Osteonecrosis of femoral head (ONFH); Osteoclasts; Antioxidant enzymes

\section{Introduction}

Osteonecrosis of the femoral head (ONFH) is a progressive degenerative disorder of the hip characterized by subchondral bone microfractures and subsequent collapse of the femoral head, eventually leading to dysfunction of the hip joint [1]. ONFH poses a huge socioeconomic burden, usually affecting young to middle aged individuals, with $\sim 67 \%$ of asymptomatic patients rapidly progressing to symptomatic stages and thus requiring total hip arthroplasties (THA) [2]. The exact aetiology of ONFH is unclear, however, steroid administration is an established risk factor, which accounts for $\sim 51 \%$ of all reported ONFH cases [3]. For example, in patients who received corticosteroid treatment for systemic 
lupus erythematosus (SLE), it was reported that an increase of corticosteroids to $10 \mathrm{mg} / \mathrm{d}$ correlated with a $3.6 \%$ increase in the rate of osteonecrosis, with daily doses $>40 \mathrm{mg}$ predisposing patients to ONFH [4]. Consistently, patients with severe acute respiratory syndrome (SARS) can also develop ONFH and progress into late stage following steroid administration [5]. The other known contributors include alcohol abuse, tobacco use, coagulation abnormalities, chemotherapy, genetic factors, and idiopathic cause [3, 6, 7].

The pathophysiologic mechanisms underlying steroid-induced $\mathrm{ONFH}$ are not fully understood. Recent studies suggest that the onset of ONFH is initiated by impaired microcirculation and necrosis of osteocytes, with magnetic resonance imaging (MRI) detecting pools of edema $[1,8]$, but without noticeable changes in subchondral bone microstructure, as assessed by computed tomography (CT). Following bone necrosis, a repair process ensues whereby osteoclast-mediated bone resorption surpasses bone formation by osteoblasts, leading to net loss of subchondral trabecular bone [9]. As the ONFH progresses, the loss of bone and altered microarchitecture compromises the integrity of the femoral head resulting in deformity and high vulnerability of collapse [8]. It is widely accepted that excessive osteoclast activity during the regenerative phase, rather than during the necrosis of cells and tissue, directly contributes to the loss of bone integrity and ensuing subchondral bone fracture [8-11]. This contribution is further supported by studies showing that anti-resorptive agents could effectively reduce the risk of femoral head collapse at early-stage ONFH when compared with placebo treatment [12-14].

A better understanding of the activation of osteoclasts during repair process is therefore critical in order to develop effective treatments to preserve the integrity of hip joint prior to femoral head deformation and collapse. Accumulated evidence indicate that reactive oxygen species (ROS) play a crucial role in osteoclast formation and function by modulating receptor activator of NF- $\mathrm{kB}$ ligand (RANKL)-induced signalling [15-17]. Our previous work has demonstrated that ROS levels are increased during estrogen deficiency-induced osteoporosis in mice, and that antioxidant agents are effective in inhibiting osteoclast activity and thus preventing bone loss [18]. ROS have also been implicated in the pathogenesis of ONFH, causing damage to blood vessels and osteoblastic cell lineages [19-21]. However, the underlying mechanisms by which ROS levels change and cause the activation of the osteoclasts in the context of steroid-induced ONFH remain poorly understood. Our study revealed that the high oxidative stress following steroid administration may result from decreased expression of antioxidant enzymes, eventually leading to osteoclast hyperactivity and subsequently ONFH progression.

\section{Methods}

\section{Specimens Collection}

Steroid-induced osteonecrotic femoral heads (ARCO stage III-IV) were collected from three patients who received total hip arthroplasty (THA) at the First Affiliated Hospital of Guangzhou University of Chinese Medicine (Guangzhou, China). All three patients with ONFH had a medical history of the steroid administration owing to different primary diseases - systemic lupus erythematosus (SLE), fever of unknown origin (FUO), and rheumatic arthritis. This study was approved by the Ethical Committee of the First Affiliated Hospital of Guangzhou University of Chinese Medicine (No. Y [2019] 141). Informed content was obtained from the patients included in this study.

\section{Steroid-induced ONFH rat Model}

Male Sprague Dawley (SD) rats at 10-weeks-of-age were provided by Sanyuanli Animal Experiment Center of Guangzhou University of Chinese Medicine (Guangzhou, China). All experimental procedures in this study were approved by the Institutional Animal Ethics Committee of Guangzhou University of Chinese Medicine (NO.20190213001). All rats were housed under a 12-hour light/dark circle and had access to water and food ad libitum. Animals were randomly divided into two groups: control group $(n=8)$ and rat ONFH model group $(\mathrm{n}=8)$. Steroid-induced ONFH models were implemented as previously described [22, 23] with modifications. Rats in the ONFH model group were given Imiquimod (IMI, $30 \mathrm{mg} / \mathrm{kg}$ ) subcutaneously on day 1 and Methylprednisolone (MPSL, $20 \mathrm{mg} / \mathrm{kg}$ ) intramuscularly on day 2 . All injections were repeated on week 4. Rats in the control group were injected with the same amount of saline at the same time point with the model group. Rats were sacrificed and the femurs were collected at 6 weeks following the first injection. Dihydroethidium (DHE, $20 \mathrm{mg} / \mathrm{kg}$ ) was subcutaneously injected 24 hours prior to sacrifice to visualize the ROS fluorescence signals of DHE [18].

\section{Histomorphometric analysis}

Rat femurs or human femoral head regions of interest were collected. The overlying tissues were removed prior to the fixation in $4 \%$ paraformaldehyde (PFA) for 48 hours. Bone tissues were decalcified in EDTA $(14 \%, \mathrm{PH}=7.4)$ (Sigma-Aldrich) at $37^{\circ} \mathrm{C}$ for 14 
days, in which the EDTA solution was changed every day till the bone became soft for sectioning. Next, the bone samples were placed in the automatic tissue processor for dehydration, followed by paraffin-embedding. Sections of 5- $\mu \mathrm{m}$ thickness were cut using a Leica RM 2155 Biocut Microtome (Leica Microsystems) and collected onto glass slides. Haematoxylin and eosin (HE) staining and tartrate-resistant acid phosphatase (TRAP) staining were implemented to visualise the bone microstructures and osteoclasts. Stained and mounted bone sections were scanned with Uscope MXII-20 (ProSciTech). Osteoclast parameters including osteoclast number per bone surface (N.Oc/BS) and osteoclast surface per bone surface (Oc.S/BS) were analysed using Bioquant Osteo software (Bioquant Image Analysis Corporation).

\section{In-vivo ROS fluorescence detection}

The cryosections of rat femoral heads were prepared as described previously [18] with modifications. Freshly isolated rat femoral heads were fixed using $4 \%$ PFA at $4{ }^{\circ} \mathrm{C}$ for 48 hours, which was followed by the decalcification process using EDTA $(0.5 \mathrm{M}, \mathrm{PH}=7.4)$ for 4 weeks at $4{ }^{\circ} \mathrm{C}$ under gentle rocking. Next, the samples were cryo-protected by incubating in solutions of $2 \%$ polyvinylpyrrolidone (PVP) and $20 \%$ sucrose for 24 hours at $4^{\circ} \mathrm{C}$. The resultant tissues were embedded in Tissue-Tek optimum cutting temperature (O.C.T.) compound (Tissue-Tech). A thickness of $5-\mu \mathrm{m}$ sections was obtained at a low speed of cutting and then collected using slides, followed by air-dry at room temperature for 30 minutes. DAPI solution was used to stain the nuclei prior to mounting using ProLong Gold Antifade Mountant (Thermo Fisher Scientific). The images were observed using an A1Si confocal microscope (NIKON). Appropriate laser settings were chosen based on the excitation/emission wavelengths of the desired fluorochrome (DAPI, 358nm/461 nm; DHE, $490 \mathrm{~nm} / 590 \mathrm{~nm}$ ) and the regions of interest were captured.

\section{Quantitative real-time polymerase chain reaction (qPCR)}

Total RNA was extracted from the bone tissue using TRIzol reagent (Life Technologies) and an RNA Extraction Kit (TaKaRa) following the manufacturer's instructions. The extracted RNA was transcribed in complementary DNA (cDNA) using reverse transcriptase with OligodT primer and moloney murine leukemia virus (MMLV) (Promega). The primers used in this study include TNFSF11, encoding receptor activator of nuclear factor kappa-B ligand (RANKL); TNFRSF11B, encoding osteoprotegerin
(OPG); CTSK, encoding cathepsin K; ACP5, encoding TRAP; CAT, encoding catalase; NQO1, encoding $\mathrm{NAD}(\mathrm{P}) \mathrm{H}$ quinone dehydrogenase 1; HMOX1, encoding heme oxygenase 1 (HO1). The primer sequences were listed in Table 1. We used SYBRGreen PCR MasterMix (Thermo Fisher Scientific) was used to perform qPCR in the ViiATM 7 Real-Time PCR System (Applied Biosystems). The qPCR data was extracted using ViiATM 7 software and the expression level of each gene was normalized to the expression of the housekeeping gene - ACTB.

Table 1. Primers sequences

\begin{tabular}{lll}
\hline Genes & Forward Sequence $\mathbf{( 5}^{\prime} \mathbf{-} \mathbf{3}^{\prime} \mathbf{)}$ & Reverse Sequence $\mathbf{( 5}^{\prime} \mathbf{s}^{\prime} \mathbf{)}$ \\
\hline TNFSF11 & CATGTTCGTGGCCCTCCTG & GGATCCATCTGCGCTCTGAA \\
TNFRSF11 & GCGCTCGTGTTCTGGACAT & ACACGGTCTTCCACTTTGCT \\
B & & \\
CTSK & GGGGGACATGACCAGTGAA & CAGAGTCTGGGGCTCTACCT \\
& G & \\
ACP5 & GGGAGATCTGTGAGCCAGTG & TTTATTCCCTCCCTGCCTGC \\
CAT & CTCCGGAACAACAGCCTTCT & ATAGAATGCCCGCACCTGAG \\
NQO1 & GCTGGTTTGAGCGAGTGTTC & CTGCCTTCTTACTCCGGAAGG \\
HMOX1 & CTGCTGACCCATGACACCAA & GGGCAGAATCTTGCACTTTGT \\
ACTB & ACAGAGCCTCGCCTTTGCC & GATATCATCATCCATGGTGAGC \\
& & TGG \\
\hline
\end{tabular}

\section{Western Blot (WB) Assay}

The bone tissues of interest were dissected and immersed in liquid nitrogen to snap freeze, which was followed by mechanical grinding using a mortar and pestle. Radioimmunoprecipitation (RIPA) lysis buffer was added and then incubated on ice for 20 minutes. The lysates were then centrifuged at 14,000 rpm for 25 minutes at $4{ }^{\circ} \mathrm{C}$. The pellets were discarded, and the supernatants were collected as protein. Protein concentrations were determined using Bicinchoninic Acid (BCA) Protein Assay Kit (Beyotime) following the manufacturer's instructions. The protein extractions were separated by SDS-polyacrylamide gel electrophoresis (SDS-PAGE), which was then transferred to nitrocellulose membrane. The membrane was blocked using 5\% skim milk (prepared in $1 \times$ TBST) for 1 hour at room temperature (RT) under slow rocking. The primary antibodies diluted in 1\% skim milk (in $1 \times$ TBST) were added to the membrane for overnight incubation at 4 ${ }^{\circ} \mathrm{C}$. On the following day, membranes were rinsed with 1XTBST (5 minutes each time for 3 times). Primary antibodies for $\beta$-actin (sc-47778), RANKL (sc-377079), cathepsin K (sc-48353), y-GCSc (sc-390811), and SOD1 (sc-101523) were purchased from Santa Cruz Biotechnology (Dallas, CA, USA). Primary antibodies for catalase (\#14097S) and HO-1 (\#70081S) were purchased from Cell Signaling Technology (Danvers, MA, USA). Appropriate anti-mouse or rabbit secondary antibodies conjugated with horseradish peroxidase (HRP) were used to 
incubate the membrane at RT for 1 hour. The proteins were detected using enhanced chemiluminescence substrate (PerkinElmer, Waltham, MA, USA). Images of protein bands were captured using an Image-quant LAS 4000 (GE Healthcare). The intensities of protein bands were analyzed using an ImageJ software (NIH, USA).

\section{Micro-CT scanning}

Rat femurs were collected, and the excess soft tissues dissected. The femoral heads were scanned in a Skyscan Micro-CT instrument (Bruker) using parameter as the following: source current, $385 \mu \mathrm{A}$; source voltage, $65 \mathrm{kV}$; pixel size $9 \mu \mathrm{m}$; filter, AI 1.0 $\mathrm{mm}$; rotation step, 0.4 degree. The image reconstruction was performed using NRecon software (Bruker) and the data was analysed using the CTAn program (Bruker). A refined volume of interest was generated $0.5 \mathrm{~mm}$ above the growth plate of the femoral head and $0.25 \mathrm{~mm}$ in height. The subchondral region of interest (ROI) within this volume was manually defined and determined using a constant threshold $(50 \sim 255)$ for binarization of the trabecular bone. The parameters of the subchondral bone, including bone volume per tissue volume (BV/TV), trabecular number (Tb.N), trabecular separation (Tb.Sp), and degree of anisotropy (DA) were compared between the control group and $\mathrm{ONFH}$ model group.

\section{Statistical Analysis}

All numeric data are presented as mean \pm standard deviation (SD). Statistical difference was determined using Student's $t$ test. The result was considered significantly different when $p$-value is less than 0.05 .

\section{Results}

\section{Imaging of late-stage of human ONFH}

The human ONFH was diagnosed using X-ray and magnetic resonance imaging (MRI). A 42-year-old female patient with SLE for 20 years was also diagnosed with renal failure for 9 years. Steroids were given orally or intravenously since SLE diagnosis in 2000. Peritoneal dialysis or haemodialysis was performed periodically. The patient reported a complaint of left hip joint pain and was then diagnosed with ONFH in 2011. ONFH was gradually progressed to a late stage which required a THA. Representative anteroposterior and frog-leg lateral radiographs of the pelvis of this patient demonstrated a crescentic lucency in the subchondral area of left femoral head (crescent sign) (Figure 1A), suggesting the separation of subchondral bone from the overlying cartilage due to collapse of the femoral head. Joint space narrowing with acetabular involvement was also clearly delineated in Figure 1A, based on which the patient was diagnosed as stage IV according to Association Research Circulation Osseous (ARCO) staging system [24]. Furthermore, MRI indicated the presence of a large-sized osteonecrotic lesion of the left femoral head, which presented as a low signal intensity area on T1-weighted image and a high signal on T2-weighted image (Figure 1B). The sequentially sliced macroscopic sections of the femoral head specimens clearly showed altered femoral head shape and osteonecrotic bone within the subchondral area (Figure 1C).

\section{Assessment of osteoclast activity and antioxidant enzymes in the necrotic bone of human femoral head}

The osteonecrotic bone tissues of the same patient were further analysed by histopathological evaluation. H\&E staining of decalcified femoral head sections showed the uniform absence of osteocytes within lacunae, indicative of osteonecrosis in the necrotic region (Figure 2A). TRAP staining revealed that the necrotic bone tissues were widely circumscribed by osteoclasts (Figure 2A). The number of osteoclasts in the necrotic region was significantly higher than that in the healthy region (Figure 2B). It is also notable that there was an increase of fat tissue accumulation in the necrotic region as compared with the healthy region (Figure 2A). To assess for osteoclast activity and explore its relationship with oxidative stress, qPCR was performed to compare the difference of associated gene expression between healthy region and necrotic region (Figure 2C). Osteoclast-specific genes, including CTSK and ACP5, were robustly up-regulated in the necrotic region compared with those in the healthy region. The ratio of TNFSF11 (encoding RANKL)/TNFRSF11B (encoding OPG) was also increased in the necrotic regions. In contrast, genes encoding anti-oxidant enzymes including CAT (encoding catalase), NQO1 (encoding $\mathrm{NAD}[\mathrm{P}] \mathrm{H}$ quinone dehydrogenase 1 ), and HMOX1 (encoding heme oxygenase 1 [HO-1]), were notably down-regulated in the necrotic bone. Furthermore, the western blot analyses of the osteoclast-related proteins (RANKL and cathepsin K) and antioxidant enzymes including catalase, $\gamma$-glutamylcysteine synthetase ( $\mathrm{\gamma}$-GCSc), and superoxide dismutase 1 (SOD1) further corroborated the mRNA findings, showing similar trends at the protein level (Figure 2D). The consistent expression trends of antioxidant enzymes and osteoclast markers were also noted in the other two cases. Together, these findings demonstrate that enhanced osteoclast 
activity is a pathological feature associated with ONFH which may, in part, due to the dysregulation of

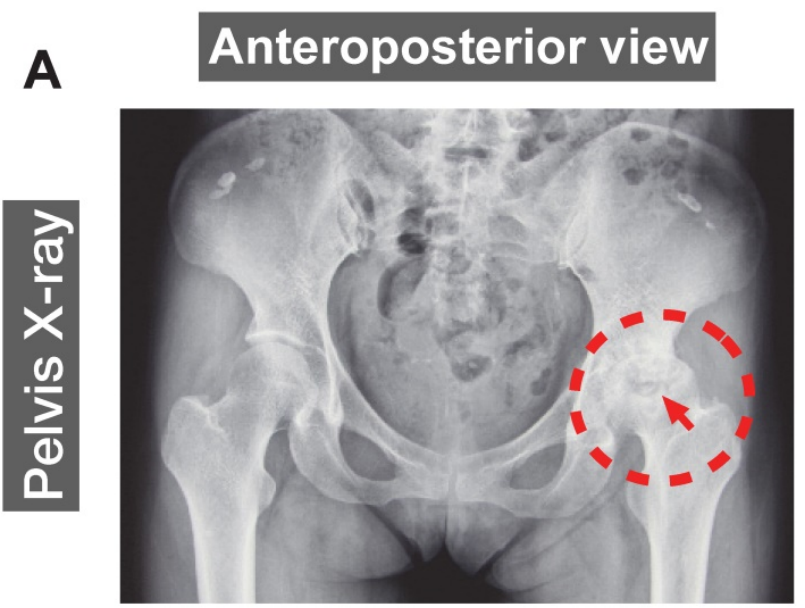

B

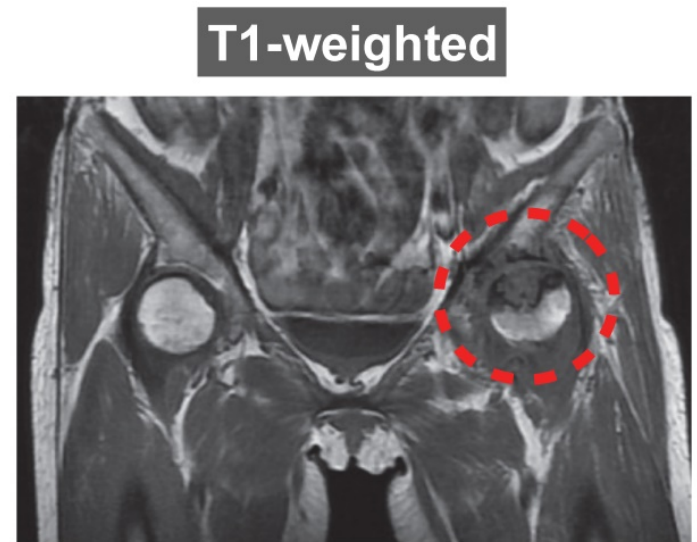

antioxidant enzymes and subsequent high oxidative stress.

\section{Frog-leg lateral view}

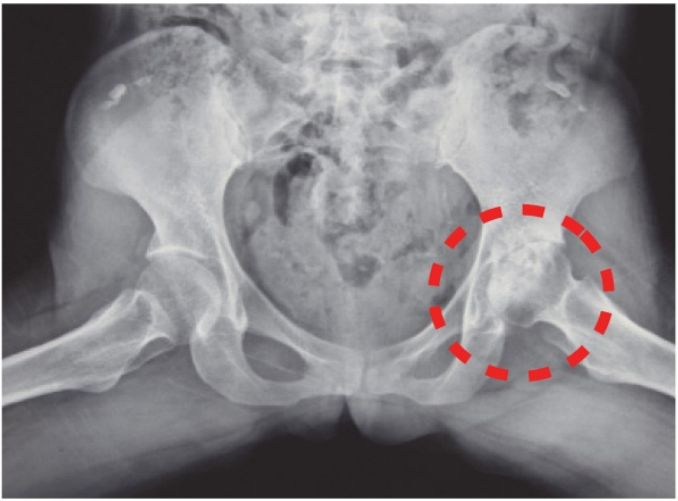

\section{T2-weighted}
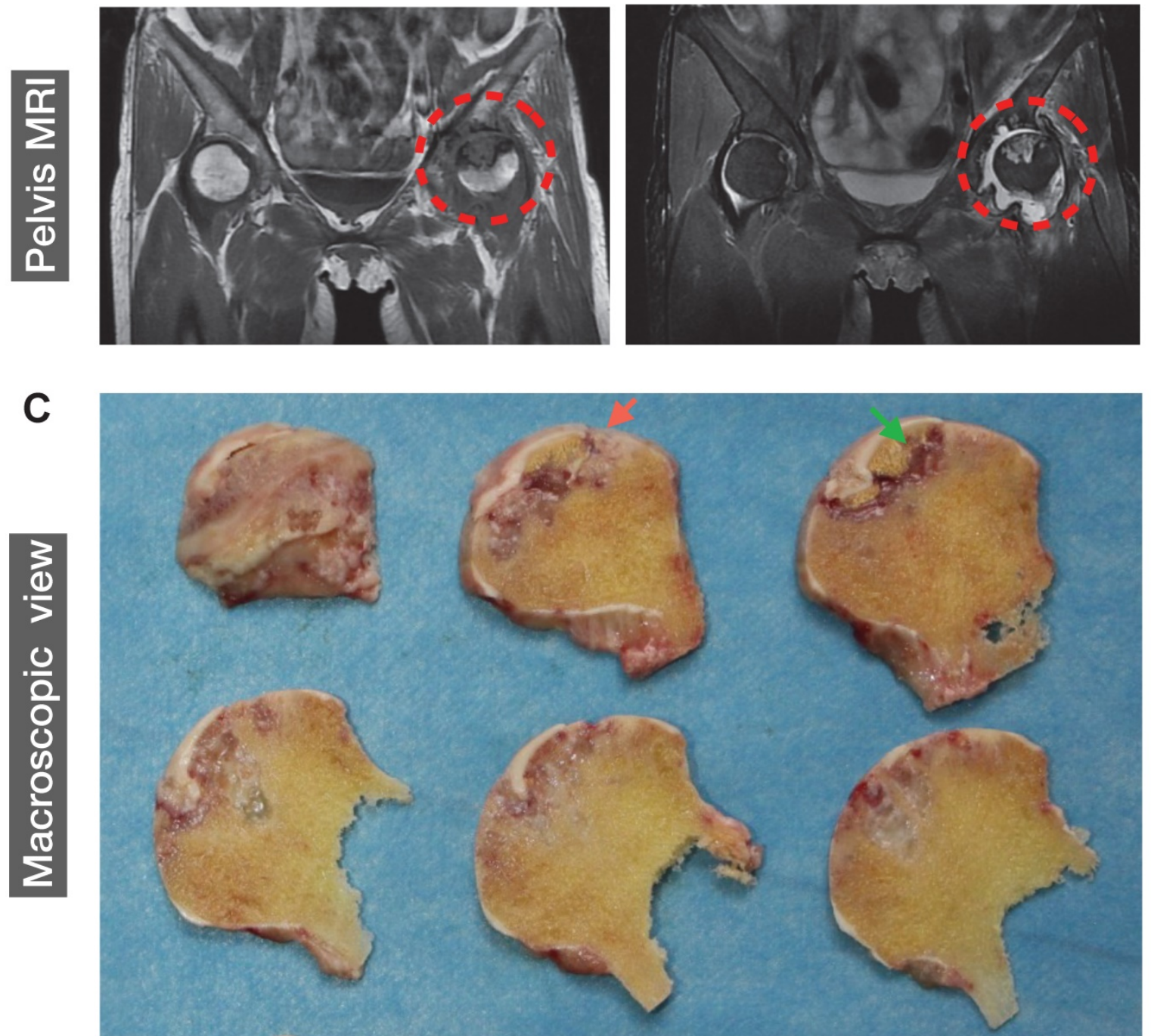

Figure 1. A representative 42-year-old female case of steroid-induced osteonecrosis of femoral head (ONFH, ARCO stage IV). (A) Radiographs delineating the crescent sign (red arrow), flattening of the articular surface of femoral head, and osteoarthritic acetabular changes (red dashed circles). (B) Coronal T1- and T2-weighted magnetic resonance images (MRI) highlighting a medium-sized necrotic lesion of the left femoral head (red dashed circles). (C) Gross appearance of sequentially sliced sections of the femoral head obtained at surgery, showing the deformation of the femoral head (red arrow) and subchondral necrotic bone (green arrow). ARCO, Association Research Circulation Osseous. 
A
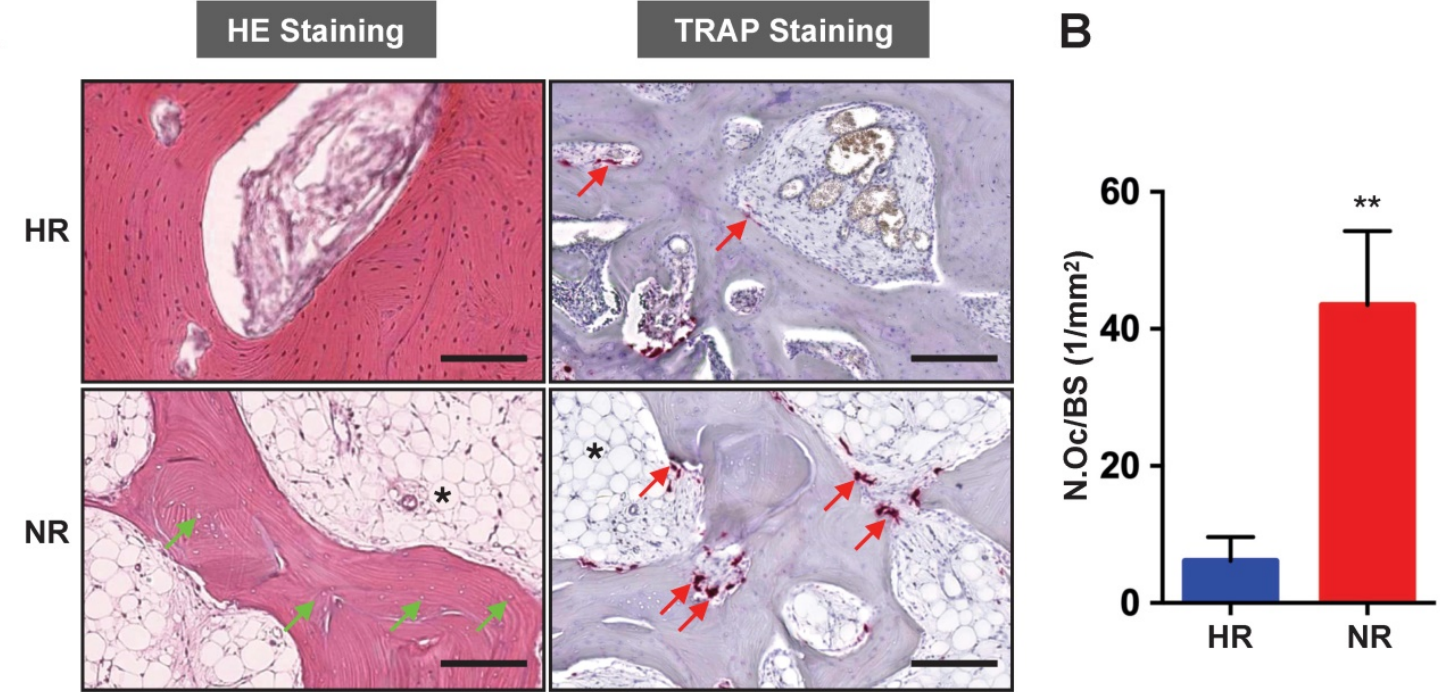

C
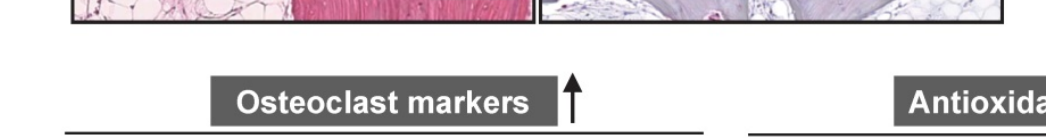

B
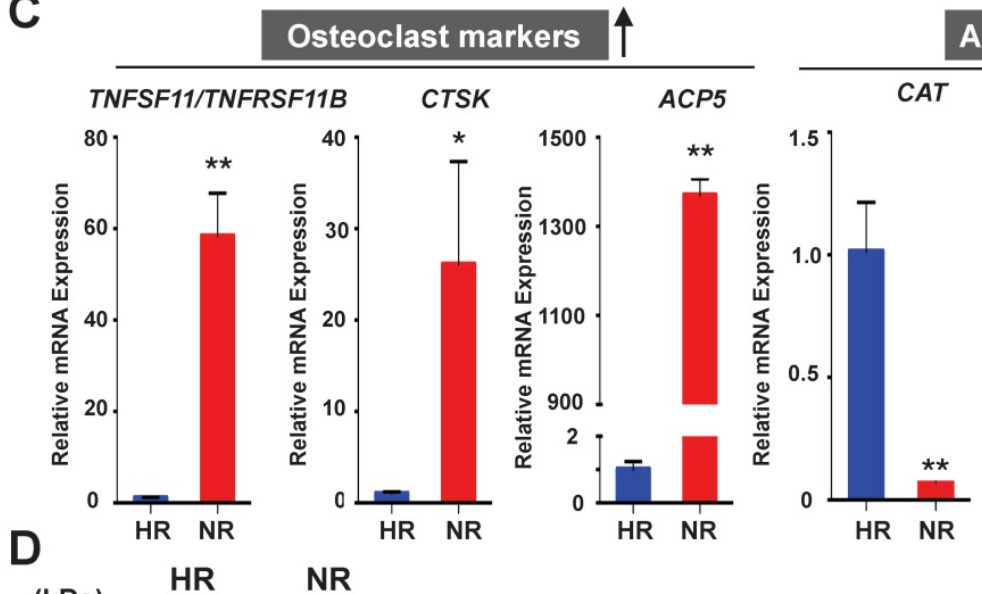

Antioxidant Enzymes $\downarrow$

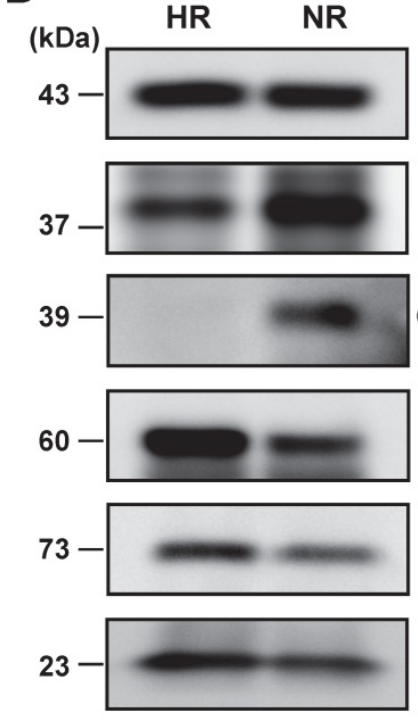

$\beta$-actin
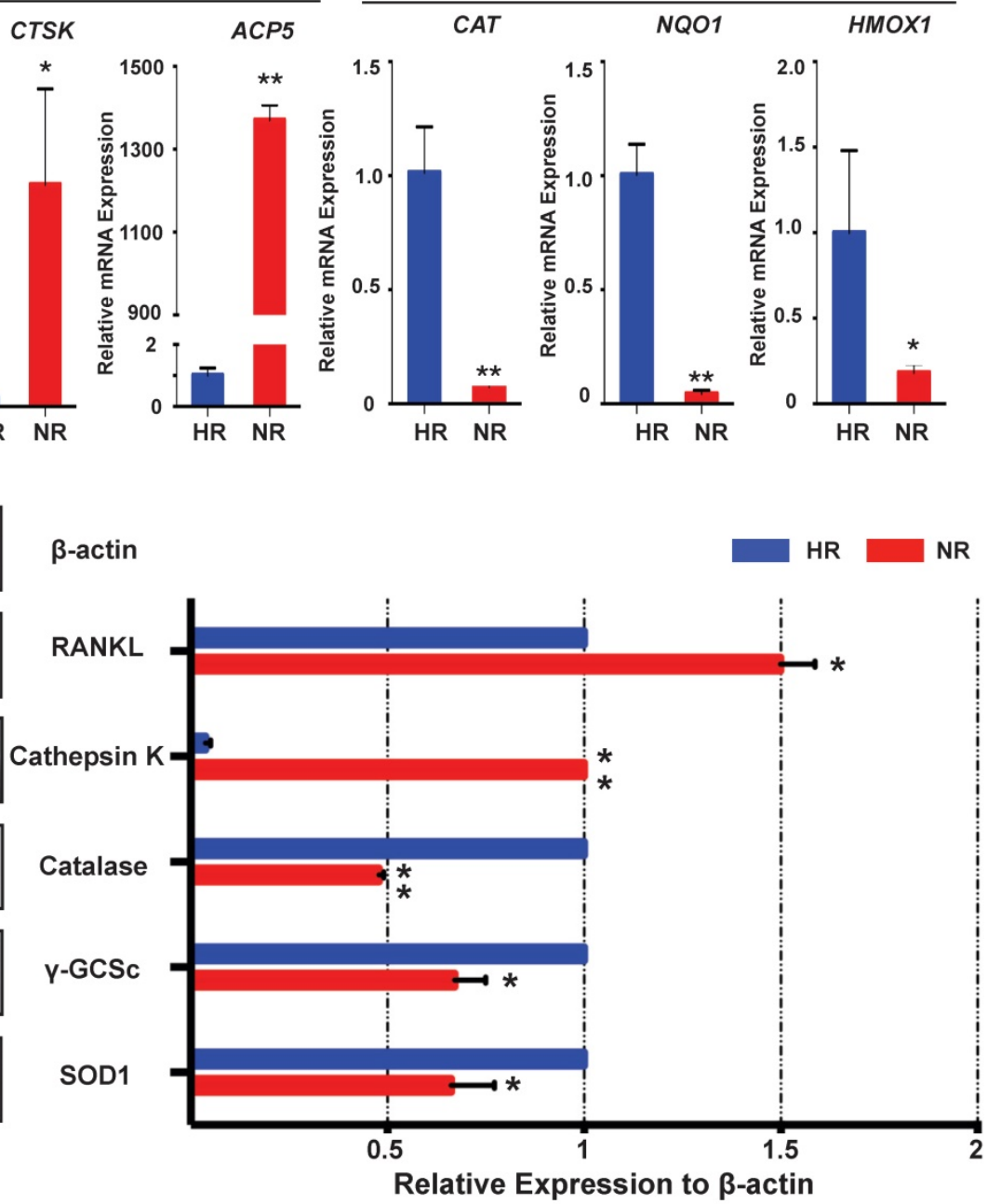

Figure 2. Necrotic area of the femoral head has enhanced osteoclast activity and decreased expression of antioxidant enzymes. (A) Hematoxylin \& Eosin (HE) staining showing the necrotic bone is featured by the presence of empty osteocyte lacunae (green arrows) and tartrate-resistant acid phosphatase (TRAP) staining showing the distributions of osteoclasts on trabecular bone surface (red arrows) in the HR and NR. The accumulation of fat tissue (asterisk) is also notable in the NR. (B) Quantitative analyses of N.OC/BS. (C) qPCR results showing the genes' expression of osteoclast-specific markers and antioxidant enzymes relative to the ACTB expression. TNFSFII, encoding receptor activator of nuclear factor kappa-B ligand (RANKL); TNFRSFI IB, encoding osteoprotegerin (OPG); CTSK, encoding cathepsin K; ACP5, encoding TRAP; CAT, encoding catalase; $\mathrm{NQO}$, encoding $\mathrm{NAD}(\mathrm{P}) \mathrm{H}$ quinone dehydrogenase $1 ; \mathrm{HMOXI}$, encoding heme oxygenase 1 (HOI). (D) Western blot analysis showing the protein expression level of osteoclast-related markers including RANKL and cathepsin $\mathrm{K}$, as well as anti-oxidant enzymes including catalase, $\mathrm{Y}$-glutamylcysteine synthetase ( $\mathrm{Y}$-GCSc), and superoxide dismutase 1 (SOD 1). All bar graphs are presented as mean $\pm \mathrm{SD}(\mathrm{n}=3$ in each group). $* \mathrm{P}<0.05, * * \mathrm{P}<0.01$ compared with control group (HR). HR, Healthy region; NR, Necrotic region; N.Oc/BS, osteoclast number/bone surface 


\section{Micro-CT based analyses of trabecular microstructure of rat femoral heads}

To further explore the relationship between osteoclasts and oxidative stress in steroid-induced $\mathrm{ONFH}$, we used a rat model to mimic ONFH (Figure 3A). The incidence rate of ONFH was 75\% (6/8) in the model group with no ONFH observed in the control group. Micro-CT was used to examine the subchondral trabecular architecture and bone structural integrity of the femoral head. As was shown in Figure 3B, the bone microstructure in the ONFH model group was notably altered following the use of steroid. Furthermore, the quantification of the parameters including BV/TV, Tb.N, and Tb.Sp in the ONFH model group were significantly inferior to those of the control group (Figure 3C), indicating the bone loss in the femoral heads of the ONFH model rats. The alteration of DA also demonstrated the interrupted bone structure following the administration of steroid (Figure 3C).

A

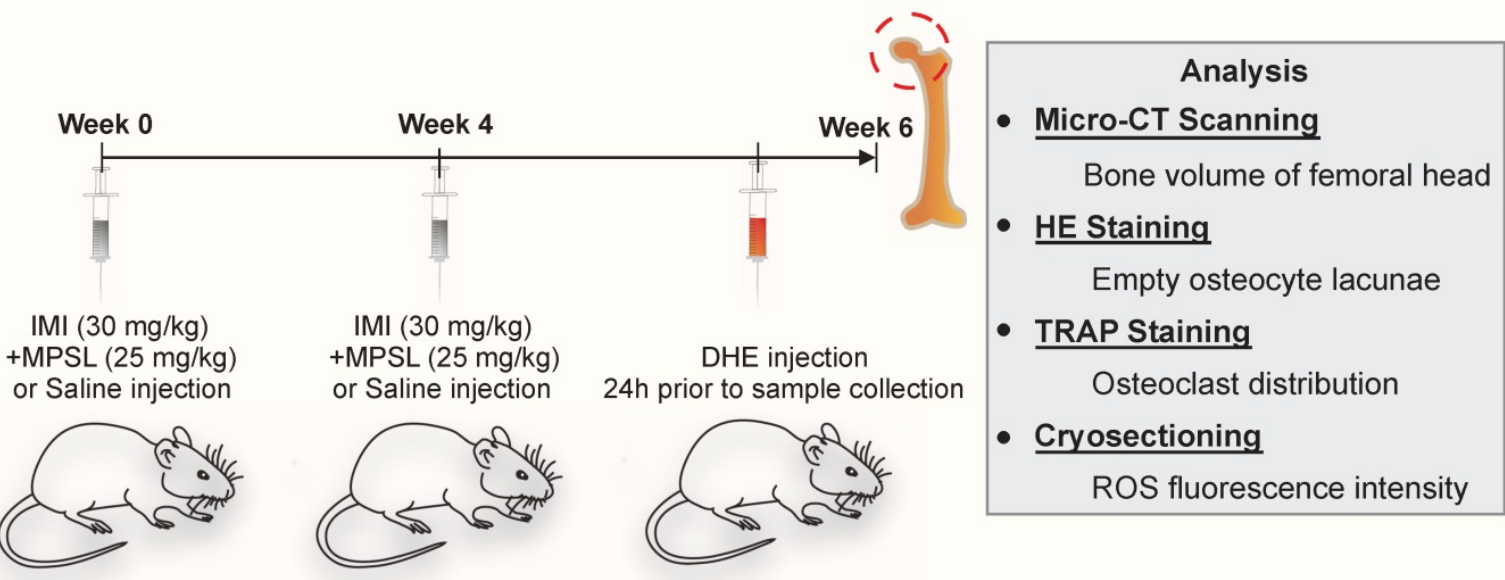

B

C
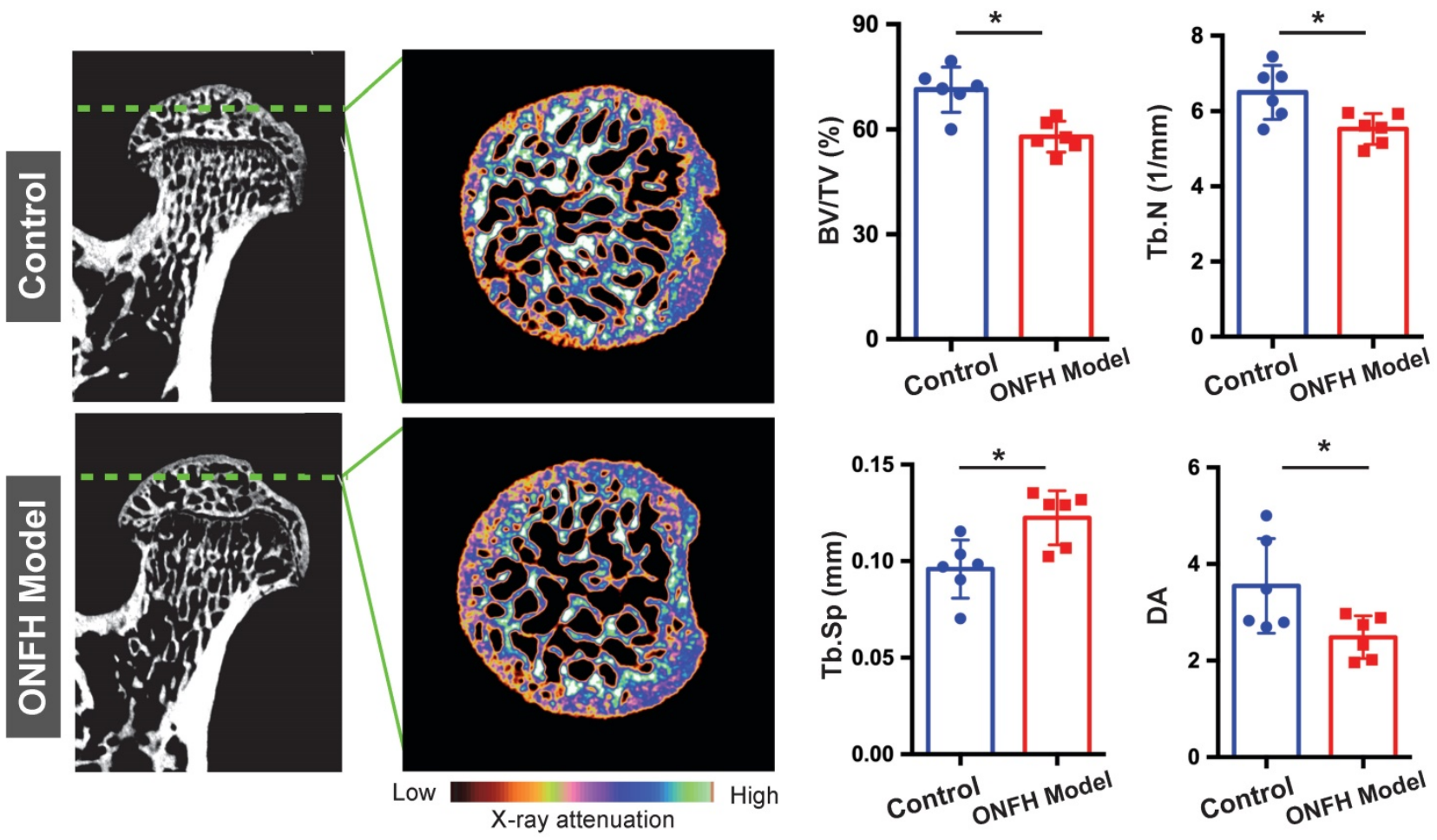

Figure 3. Steroid-induced osteonecrosis of femoral head (ONFH) of rat and micro-CT ( $\mu$ CT) analysis. (A) Schematic illustration of the establishment of rat ONFH model. (B) Representative $\mu$ CT scanning images of the femoral head of the control group and model group. (C) Quantification of the bone microstructure parameters of the femoral head, including bone volume/tissue volume (BV/TV), trabecular number (Tb.N), trabecular separation (Tb.Sp), degree of anisotropy (DA). All bar graphs are presented as mean $\pm S D(n=6$ in each group); $* P<0.05$ compared with the control group; $n s$, no significance. 


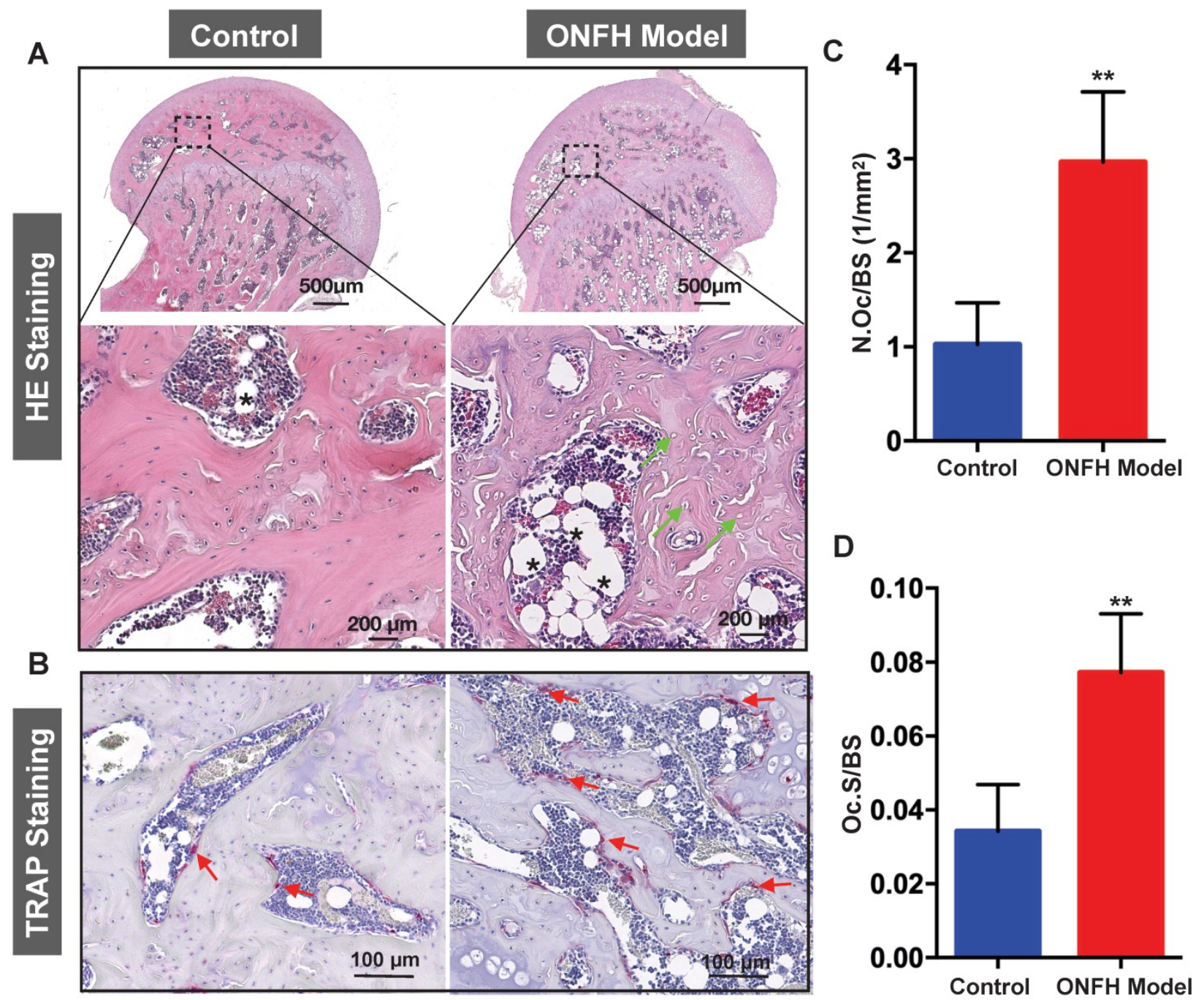

Figure 4. Histomorphometric analysis of steroid-induced osteonecrosis of femoral head (ONFH) in rat model. (A) Hematoxylin \& Eosin (HE) staining of decalcified paraffin-embedding sections showing the increased number of empty lacunae (green arrows) and adipose tissue area (asterisk) in the model group. (B) Tartrate-resistant acid phosphatase (TRAP) staining showing the osteoclasts (red arrows) in the control and model groups. (C and D) Quantitative analyses of N.OC/BS and Oc.S/BS (N=5 per group). **P<0.01 compared with control group. N.Oc/BS, osteoclast number/bone surface; Oc.S/BS, osteoclast surface/bone surface.

\section{Histomorphometric analysis of steroid induced ONFH rat model}

To complement the studies, histomorphometric analyses were performed on decalcified sections of the femoral heads. From the H\&E staining (Figure 4A), increased bone marrow adiposity and the presence of empty osteocyte lacunae were observed in the subchondral bone tissues, reminiscent of histopathological features observed in patient samples with ONFH. In addition, TRAP staining revealed a morphological increase in both osteoclast number (Figure 4B and C) and osteoclast surface (Figure 4B and D) surrounding the bone samples following steroid administration when compared with the osteoclasts in the control group.

\section{Detection of ROS and osteoclast-related markers in the rat femoral heads}

The ex vivo ROS level, as probed by DHE, was detected in the cryosections of femoral head under the confocal microscopy. As was shown in Figure 5A, DHE fluorescence of the subchondral area of the femoral head was dramatically enhanced in the model group. The fluorescence intensity profile of the yellow indicated line in the Figure $\mathbf{5 A}$ showed a clear difference between the control group and model group (Figure 5B). Quantification of the DHE fluorescence intensity indicated a higher oxidative stress in the rat femoral head following the steroid administration (Figure 5C). Western blot assay of the femoral head tissues further indicated that the expression levels of antioxidant enzymes such as HO-1, catalase, and SOD1 were significantly down-regulated in the model group (Figure 5D). In 
contrast, the expression of the mature osteoclast marker cathepsin $\mathrm{K}$ was upregulated in the ONFH model group (Figure 5D). In addition, the expression of RANKL was also increased (Figure 5D). Collectively, these results suggest that the use of steroid may lead to high oxidative stress in the femoral head by reducing antioxidant enzymes, which may contribute to the recruitment and/or activation of osteoclasts.
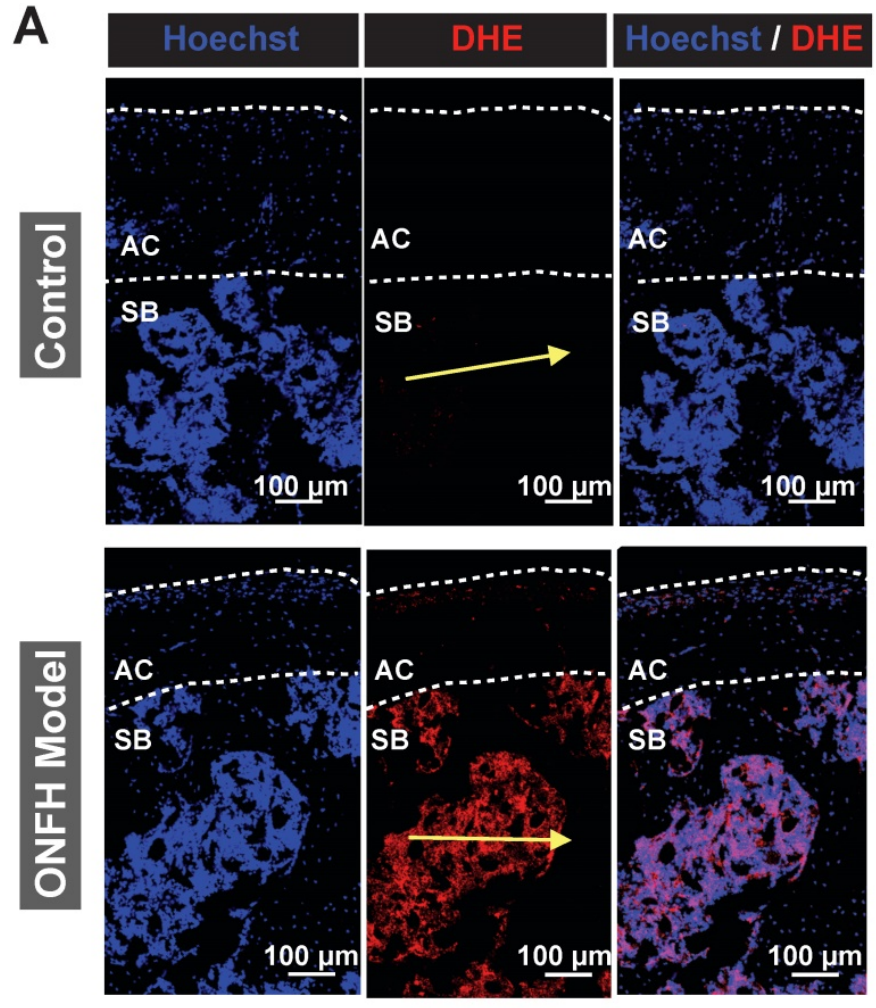

B

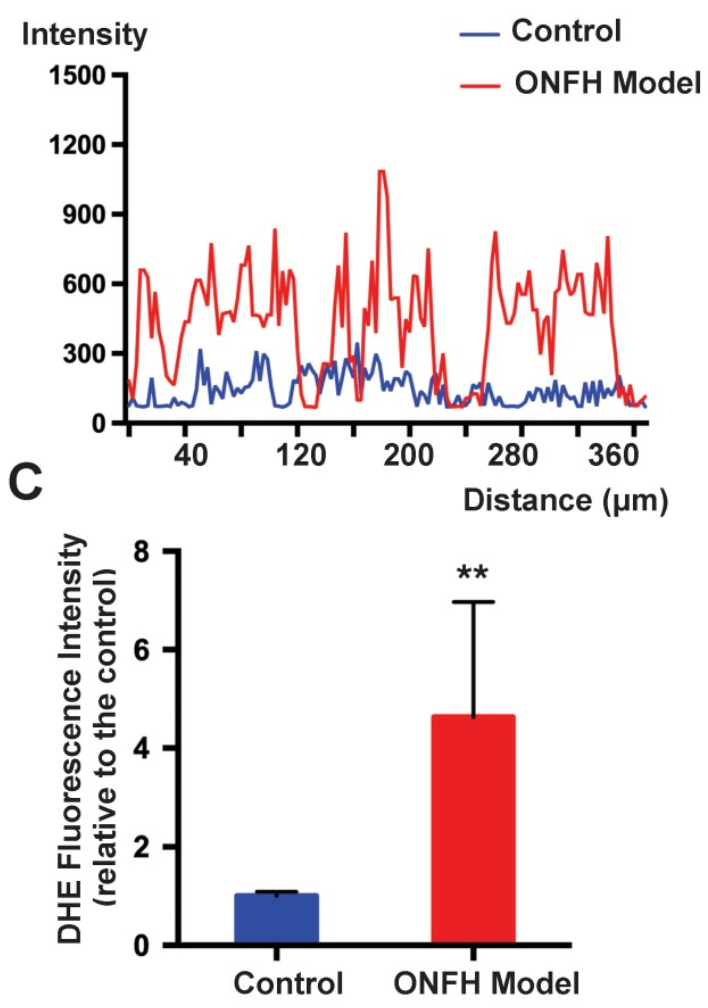

D
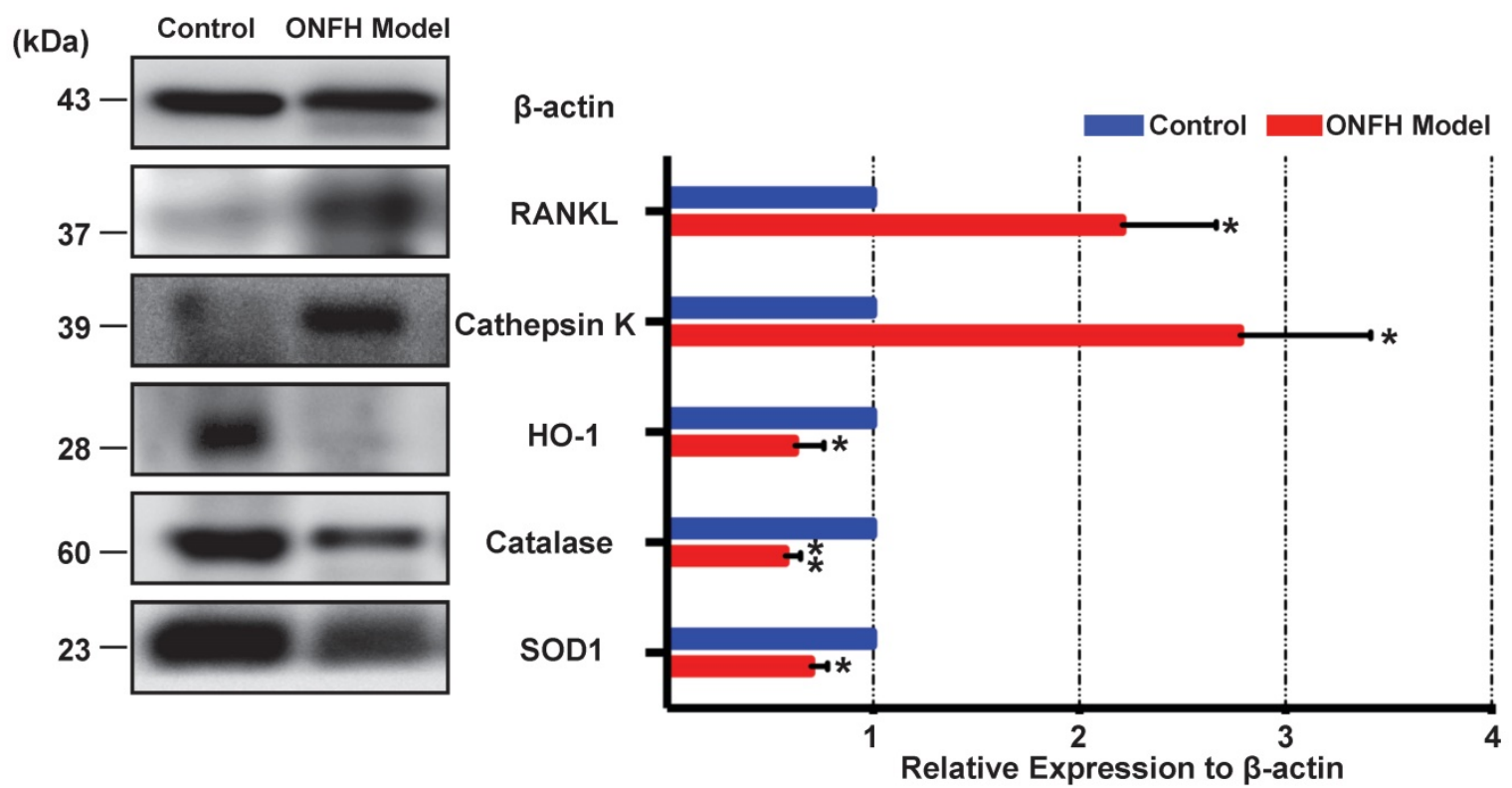

Figure 5. Analysis of reactive oxygen species (ROS) level and antioxidant enzymes in the rat femoral head. (A) Cryosections of femoral heads showing ROS level, as probed by dihydroethidium (DHE), in the control group and model group. AC, articular cartilage (white dashed line area); SB, subchondral bone. (B) DHE fluorescence intensity profile through subchondral area of femoral head (indicated by the yellow arrow in A) The abscissa of the DHE intensity profile represents the distance from the "start point" of the yellow arrow. (C) Quantification of DHE fluorescence intensity relative to the control group ( $n=5$ in each group). (D) Western blot analysis of the protein expression level of osteoclast-related markers including RANKL and cathepsin $\mathrm{K}$, as well as anti-oxidant enzymes including heme oxygenase 1 (HO1), catalase, and superoxide dismutase 1 (SOD1) ( $\mathrm{n}=3$ in each group). All bar graphs are presented as mean \pm SD. $* P<0.05, * * P<0.01$ compared with control group. 


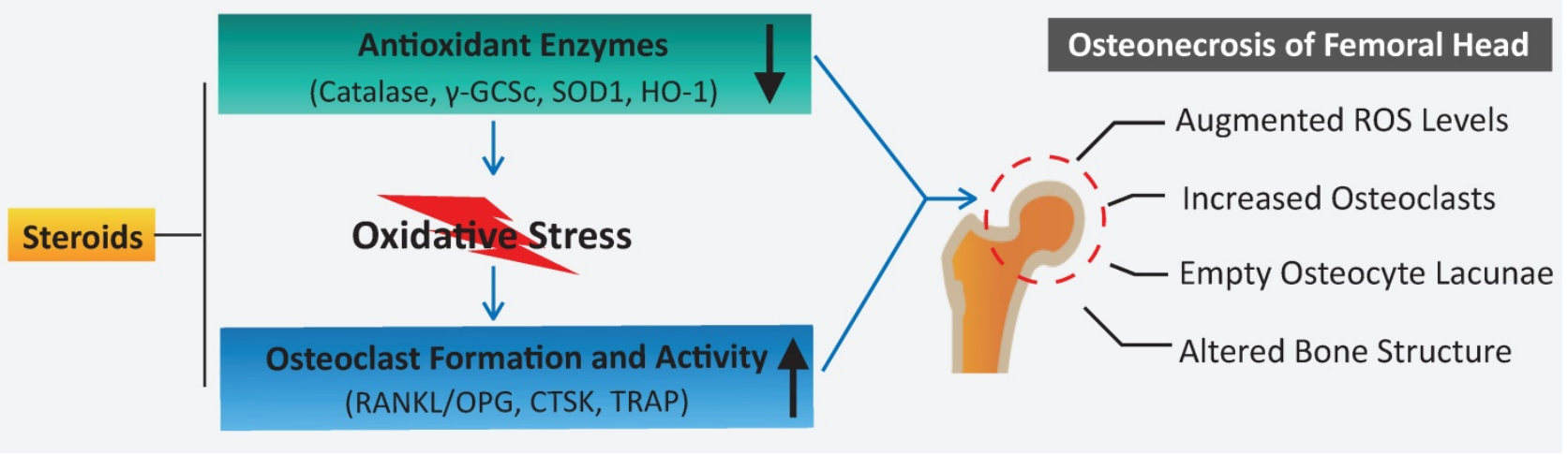

Figure 6. A proposed scheme for enhanced oxidative stress and hyperactive osteoclasts following the use of steroids. A decline in the expression of antioxidant enzymes correlates with the administration of steroids, which may, in turn, contribute to elevated ROS levels and subsequent osteoclast numbers/activity observed during the pathogenesis and progression of ONFH. HOI: heme oxygenase 1; ROS: reactive oxygen species; RANKL: receptor activator of NF-KB ligand; TRAP: tartrate-resistant acid phosphatase; $\mathrm{Y}$-GCSc: Y-glutamylcysteine synthetase.

\section{Discussion}

The use of steroids, particularly the prolonged and high-dose administration, is estimated to account for the majority of non-traumatic ONFH cases [4]. $\mathrm{ONFH}$ is a progressive bone disorder which typically results in the collapse of the femoral head at end stage. The precise cellular and molecular mechanisms underlying steroid-induced osteonecrosis remain controversial. Recent studies indicate that increased osteoclast number and activity are responsible for the femoral head bone loss and subsequent failure $[8,10$, 11]. Given the increasing number of studies illustrating a role promising or ROS in promoting osteoclast formation and resorption, we sought to elucidate whether alterations of ROS level and osteoclasts lead to the pathogenesis of ONFH following the use of steroids. In the present study, ROS-related enzymes and osteoclasts were detected in both patients' ONFH specimens and rodent models mimicking ONFH. Specimens of femoral head were obtained from late-stage patients with progressive collapse of femoral head and required THA. Antioxidant enzymes and osteoclasts were then compared between healthy and necrotic regions. Steroid-induced ONFH rat models were then established to further assess the role of ROS and osteoclasts that played in this bone disorder.

Oxidative stress is featured by an augmented level of ROS which disrupts physiological reduction-oxidation (redox) balance. ROS are known to play a pivotal role in aging and degenerative diseases $[25,26]$. The deleterious effects of ROS result from the alternations to the integrity of mitochondrial and nuclear DNA, which can lead to cell apoptosis or necrosis [27]. Accumulating studies have suggested that bone pathophysiology is closely related with redox balance and ROS are key modulators of bone cell functions [28]. High oxidative stress is thought to be associated with the development of various bone disorders, including osteoporosis [29, 30], bone tumours [31], diabetic osteopenia [32], rheumatoid arthritis [33], and ankylosing spondylitis [34]. Recent evidence also indicated that ROS are involved in the pathogenesis of steroid-induced ONFH [20, 21, 35] and that approaches inhibiting oxidative stress exert potential therapeutic effects on ONFH [35-37]. To validate this, a ROS probe (DHE) was administrated on the rats prior to sacrifice, which is distributed via the circulation enabling the direct visualization of ROS in tissues as we previously described [18]. Not surprisingly, the ROS fluorescence intensity in the microenvironment of subchondral area in femoral head was dramatically enhanced following steroid treatment. This is, to the best of our knowledge, the first time that the ROS levels have been directly visualized in the femoral head ex vivo. However, we are unable to identify the specific source of ROS, which may also be partly contributed by the altered conditions of osteocytes and fat tissues.

Oxidative stress is determined by the imbalance between ROS generation and its scavenging, the latter of which is carried out by well-known antioxidant enzymes such as SOD, HO- 1 , catalase, and $\gamma$-GCSc [38]. The mechanism underlying the steroid-induced oxidative stress in the femoral head [39] remains unclear, but is thought that the administration of steroid leads to the downregulation of cytoprotective enzymes and subsequently causes redox failure [40, 41]. Indeed, our results confirmed that the antioxidant enzymes (catalase, $\gamma$-GCSc, and SOD1) were significantly altered in the necrotic area of patients' femoral head relative to the healthy area, suggesting the existence of redox failure and the high level of ROS in the necrotic area. SOD plays a major role in 
cellular redox by reducing superoxide radicals to hydrogen peroxide $\left(\mathrm{H}_{2} \mathrm{O}_{2}\right)$ which is less unstable [38]. Catalase then converts $\mathrm{H}_{2} \mathrm{O}_{2}$ into oxygen and water, thereby protecting the cells from ROS damage [42]. Furthermore, in addition to high ROS signalling in the femoral head, rats received steroids injection also showed decreased expressions of antioxidant enzymes including HO-1, catalase, and SOD1. A previous study demonstrated that the steroid administration may affect the antioxidant enzymes site-specifically, which was featured by an increased expression level of antioxidant enzymes such as SOD and catalase in liver, kidney, and heart as compared with humerus and femur [40]. Following the use of steroid, the oxidation-reduction homeostasis in bone is compromised and therefore the bone was thought to be more susceptible to oxidative stress. These findings collectively suggest that the compromised expressions of antioxidant enzymes are, at least partly, responsible for the oxidative stress due to steroid administration.

The current understanding of the hazards of ROS is that oxidative stress may lead to pathological conditions, including the elevated vascular permeability [43] as well as DNA oxidation injuries [39] which cause cell apoptosis or necrosis [37]. However, whether ROS enhance the osteoclast activity during the pathogenesis of ONFH has not been previously reported. ROS can support the activation of RANKL-induced signalling, which is essential for osteoclast formation and resorptive activity $[15,18,44]$. The exact molecules or proteins that ROS target remain unclear, but RANKL-induced downstream events such as MAPK [15] and NF-кB signaling [45] appear to be involved. Our results demonstrate that the reduced antioxidant enzymes in the necrotic area are accompanied by the augmented osteoclast-related markers, such as cathepsin K, which were also supported by the TRAP staining indicating that osteoclasts activity is localized to the necrotic area. However, more clinical specimens as well as the investigation of mechanisms underlying the declined expression of the antioxidant enzymes are still needed in further study. Given the increased accumulation of fat tissue in the necrotic region, the loss of bone structure integrity could also be partly due to the impaired osteoblast differentiation. The corticosteroid treatment could increase adipogenesis by activating peroxisome proliferator-activated receptor gamma (PPAR $\gamma)$, but decrease osteogenesis, which was well documented previously [36, 46, 47]. Consistent with these human specimens, we found that osteoclast numbers were elevated in rat models of steroid-induced ONFH, which ultimately resulted in decreased femoral trabecular bone volume as assessed by $\mu \mathrm{CT}$ (BV/TV, Tb.N, Tb.Sp). Relative to the control group, rats of the ONFH group also showed structurally disorganized bone as examined by the measurement of anisotropy. These results indicate that oxidative stress is closely related with the osteoclast activity and thus may coincide with the rapid progression of ONFH.

However, there are also some limitations in this study. Firstly, an anti-ROS group was not included in this study. It was previously indicated that anti-oxidative stress treatments indeed prevent or alleviate steroid-induced ONFH by affecting blood vessel, osteocytes, and adipose tissues [35, 36, 48-50], but with the lack of data on the role of osteoclasts. Osteoclast acts as a major contributor to the collapse of femoral head and the agents like bisphosphonates were also shown to have beneficial effects on ONFH [13]. Herein, anti-ROS agents may also exhibit therapeutic effects by inhibiting excessive osteoclasts in steroid-induced ONFH, which needs to be further determined. Secondly, the outcomes or pathogenesis of steroid-induced ONFH in animals of different species [51] or/and in human may be variable. The human samples were collected from late-stage ONFH, while the animal models used in this study were more likely to be at early stage which may not coincide with the results on clinical perspective. Currently, rat models are still considered as desirable and suitable preclinical models to study molecular mechanisms of metabolic diseases [46]. In this study, we proposed that ROS may activate osteoclasts and subsequently lead to the progression of ONFH. To address this hypothesis, early-stage rat ONFH seems well-represent the steroid-induced alterations of ROS and osteoclasts before the femoral head collapse. On the other hand, given the hard access to the human specimens at early stage, more advanced animal models which can mimic the late-stage ONFH are still required to be developed in the future.

In summary, this study demonstrates that a decline in the expression of antioxidant enzymes correlates with the administration of steroids, which may, in turn, contribute to elevated ROS levels and osteoclast numbers/activity observed during the pathogenesis of ONFH. Oxidative injuries usually present shortly following steroid administration at the very early-stage ONFH [39, 48], and excessive osteoclast activity is also believed to be a contributor for femoral head collapse. This provides rationale that antioxidant therapy may be a promising alternative to prevent the progression or collapse of steroid-induced ONFH by suppressing ROS level and thus inhibiting osteoclasts. 


\section{Abbreviations}

ARCO: Association Research Circulation Osseous; BCA: Bicinchoninic Acid; BV/TV: bone volume per tissue volume; CAT: catalase; CT: computed tomography; DA: degree of anisotropy; DHE: dihydroethidium; HO1: heme oxygenase 1; IMI: imiquimod; MMLV: moloney murine leukemia virus; MPSL: methylprednisolone; MRI: magnetic resonance imaging; NQO1: NAD[P]H quinone dehydrogenase 1; OCT: optimum cutting temperature; ONFH: osteonecrosis of the femoral head; OPG: osteoprotegerin; PFA: paraformaldehyde; PVP: polyvinylpyrrolidone; qPCR: Quantitative real-time polymerase chain reaction; ROS: reactive oxygen species; RANKL: receptor activator of NF-KB ligand; RIPA: radioimmunoprecipitation; SLE: systemic lupus erythematosus; SOD1: superoxide dismutase 1; THA: total hip arthroplasty; Tb.N: trabecular number; Tb.Sp: trabecular separation; TRAP: tartrate-resistant acid phosphatase; $Y$-GCSc: $\gamma$-glutamylcysteine synthetase.

\section{Acknowledgements}

This study was supported in part by grants from the Australian Health and Medical Research Council (NHMRC, No. 1107828, 1127156, 1163933). This study was also supported by the Inheritance Studio Construction Project of Prestigious TCM Doctors (Wei $\mathrm{He}$ ) of Guangdong Province (No. YZYBH [2017]17) and Excellent Doctoral Dissertation Incubation Grant of Guangzhou University of Chinese Medicine (No. GZYY [2018]62). Kai Chen is supported by Australian Government Research Training Program Scholarship. Yuhao Liu was sponsored by $\mathrm{PhD}$ scholarships under the State Scholarship Fund from China Scholarship Council (No. LJF [2018]3101/201808440486) and Postgraduate Exchange Program from Guangzhou University of Chinese Medicine (No. GZYY [2016]83). We acknowledge the facilities and technical assistance of the Centre for Microscopy, Characterization \& Analysis, the University of Western Australia.

\section{Competing Interests}

The authors have declared that no competing interest exists.

\section{References}

1. Guerado E, Caso E. The physiopathology of avascular necrosis of the femoral head: an update. Injury. 2016; 47 Suppl 6: S16-S26.

2. Musso ES, Mitchell SN, Schink-Ascani M, Bassett CA. Results of conservative management of osteonecrosis of the femoral head. A retrospective review. Clin Orthop Relat Res. 1986: 209-15.

3. Fukushima W, Fujioka M, Kubo T, Tamakoshi A, Nagai M, Hirota Y. Nationwide epidemiologic survey of idiopathic osteonecrosis of the femoral head. Clin Orthop Relat Res. 2010; 468: 2715-24.

4. Mont MA, Pivec R, Banerjee S, Issa K, Elmallah RK, Jones LC. High-Dose Corticosteroid Use and Risk of Hip Osteonecrosis: Meta-Analysis and Systematic Literature Review. J Arthroplasty. 2015; 30: 1506-12 e5.
5. Zhao FC, Guo KJ, Li ZR. Osteonecrosis of the femoral head in SARS patients: seven years later. Eur J Orthop Surg Traumatol. 2013; 23: 671-7.

6. Wei QS, Hong GJ, Yuan YJ, Chen ZQ, Zhang QW, He W. Huo Xue Tong Luo capsule, a vasoactive herbal formula prevents progression of asymptomatic osteonecrosis of femoral head: A prospective study. J Orthop Translat. 2019; 18: 65-73.

7. Mont MA, Cherian JJ, Sierra RJ, Jones LC, Lieberman JR. Nontraumatic Osteonecrosis of the Femoral Head: Where Do We Stand Today? A Ten-Year Update. J Bone Joint Surg Am. 2015; 97: 1604-27.

8. Weinstein RS, Hogan EA, Borrelli MJ, Liachenko S, O'Brien CA, Manolagas SC. The Pathophysiological Sequence of Glucocorticoid-Induced Osteonecrosis of the Femoral Head in Male Mice. Endocrinology. 2017; 158: 3817-31.

9. Shah KN, Racine I, Jones LC, Aaron RK. Pathophysiology and risk factors for osteonecrosis. Curr Rev Musculoskelet Med. 2015; 8: 201-9.

10. Wang C, Meng H, Wang Y, Zhao B, Zhao C, Sun W, et al. Analysis of early stage osteonecrosis of the human femoral head and the mechanism of femoral head collapse. Int J Biol Sci. 2018; 14: 156-64.

11. Samara S, Dailiana Z, Chassanidis C, Koromila T, Papatheodorou L, Malizos $\mathrm{KN}$, et al. Expression profile of osteoprotegerin, RANK and RANKL genes in the femoral head of patients with avascular necrosis. Exp Mol Pathol. 2014; 96: 9-14.

12. Kim HK, Randall TS, Bian H, Jenkins J, Garces A, Bauss F. Ibandronate for prevention of femoral head deformity after ischemic necrosis of the capital femoral epiphysis in immature pigs. J Bone Joint Surg Am. 2005; 87: 550-7.

13. Lai KA, Shen WJ, Yang CY, Shao CJ, Hsu JT, Lin RM. The use of alendronate to prevent early collapse of the femoral head in patients with nontraumatic osteonecrosis. A randomized clinical study. J Bone Joint Surg Am. 2005; 87: 2155-9.

14. Hofstaetter JG, Wang J, Yan J, Glimcher MJ. The effects of alendronate in the treatment of experimental osteonecrosis of the hip in adult rabbits. Osteoarthritis Cartilage. 2009; 17: 362-70.

15. Lee NK, Choi YG, Baik JY, Han SY, Jeong DW, Bae YS, et al. A crucial role for reactive oxygen species in RANKL-induced osteoclast differentiation. Blood. 2005; 106: 852-9.

16. Kim H, Lee YD, Kim HJ, Lee ZH, Kim HH. SOD2 and Sirt3 Control Osteoclastogenesis by Regulating Mitochondrial ROS. J Bone Miner Res. 2017; 32: 397-406.

17. Yip KH, Zheng MH, Steer JH, Giardina TM, Han R, Lo SZ, et al. Thapsigargin modulates osteoclastogenesis through the regulation of RANKL-induced signaling pathways and reactive oxygen species production. J Bone Miner Res. 2005; 20: 1462-71.

18. Chen K, Qiu P, Yuan Y, Zheng L, He J, Wang C, et al. Pseurotin A Inhibits Osteoclastogenesis and Prevents Ovariectomized-Induced Bone Loss by Suppressing Reactive Oxygen Species. Theranostics. 2019; 9: 1634-50.

19. Ichiseki T, Kaneuji A, Ueda Y, Nakagawa S, Mikami T, Fukui K, et al. Osteonecrosis development in a novel rat model characterized by a single application of oxidative stress. Arthritis Rheum. 2011; 63: 2138-41.

20. Tong $\mathrm{P}, \mathrm{Wu} \mathrm{C}$, Jin $\mathrm{H}, \mathrm{Mao} \mathrm{Q}, \mathrm{Yu} \mathrm{N}, \mathrm{Holz} \mathrm{JD}$, et al. Gene expression profile of steroid-induced necrosis of femoral head of rats. Calcif Tissue Int. 2011; 89: 271-84.

21. Ichiseki $\mathrm{T}$, Ueda $\mathrm{Y}$, Katsuda $\mathrm{S}$, Kitamura $\mathrm{K}$, Kaneuji A, Matsumoto $\mathrm{T}$. Oxidative stress by glutathione depletion induces osteonecrosis in rats. Rheumatology (Oxford). 2006; 45: 287-90.

22. Okazaki S, Nagoya $S$, Matsumoto $H$, Mizuo K, Sasaki M, Watanabe $S$, et al. Development of non-traumatic osteonecrosis of the femoral head requires toll-like receptor 7 and 9 stimulations and is boosted by repression on nuclear factor kappa B in rats. Lab Invest. 2015; 95: 92-9.

23. Jiang $Y$, Zhang $Y$, Chen W, Liu C, Li X, Sun D, et al. Achyranthes bidentata extract exerts osteoprotective effects on steroid-induced osteonecrosis of the femoral head in rats by regulating RANKL/RANK/OPG signaling. J Transl Med. 2014; 12: 334.

24. Yoon BH, Mont MA, Koo KH, Chen CH, Cheng EY, Cui Q, et al. The 2019 Revised Version of Association Research Circulation Osseous Staging System of Osteonecrosis of the Femoral Head. J Arthroplasty. 2019; 35:933-40.

25. Krause KH. Aging: a revisited theory based on free radicals generated by NOX family NADPH oxidases. Exp Gerontol. 2007; 42: 256-62.

26. Valko M, Leibfritz D, Moncol J, Cronin MT, Mazur M, Telser J. Free radicals and antioxidants in normal physiological functions and human disease. Int J Biochem Cell Biol. 2007; 39: 44-84.

27. Naka K, Muraguchi T, Hoshii T, Hirao A. Regulation of reactive oxygen species and genomic stability in hematopoietic stem cells. Antioxid Redox Signal. 2008; 10: 1883-94.

28. Wauquier F, Leotoing L, Coxam V, Guicheux J, Wittrant Y. Oxidative stress in bone remodelling and disease. Trends Mol Med. 2009; 15: 468-77.

29. Sendur OF, Turan Y, Tastaban E, Serter M. Antioxidant status in patients with osteoporosis: a controlled study. Joint Bone Spine. 2009; 76: 514-8.

30. Callaway DA, Jiang JX. Reactive oxygen species and oxidative stress in osteoclastogenesis, skeletal aging and bone diseases. J Bone Miner Metab. 2015; 33: 359-70

31. Magwere T, Myatt SS, Burchill SA. Manipulation of oxidative stress to induce cell death in Ewing's sarcoma family of tumours. Eur J Cancer. 2008; 44: 2276-87. 
32. Hamada Y, Kitazawa S, Kitazawa R, Fujii H, Kasuga M, Fukagawa M. Histomorphometric analysis of diabetic osteopenia in streptozotocin-induced diabetic mice: a possible role of oxidative stress. Bone. 2007; 40: 1408-14.

33. Kabuyama Y, Kitamura T, Yamaki J, Homma MK, Kikuchi S, Homma Y. Involvement of thioredoxin reductase 1 in the regulation of redox balance and viability of rheumatoid synovial cells. Biochem Biophys Res Commun. 2008; 367: 491-6.

34. Karkucak M, Capkin E, Alver A, Akyuz A, Kiris A, Ak E, et al. The effect of anti-TNF agent on oxidation status in patients with ankylosing spondylitis. Clin Rheumatol. 2010; 29: 303-7.

35. Jia $Y B$, Jiang DM, Ren $Y Z$, Liang $Z H$, Zhao ZQ, Wang $Y X$. Inhibitory effects of vitamin $\mathrm{E}$ on osteocyte apoptosis and DNA oxidative damage in bone marrow hemopoietic cells at early stage of steroid-induced femoral head necrosis. Mol Med Rep. 2017; 15: 1585-92.

36. Zhang G, Qin L, Sheng H, Wang XL, Wang YX, Yeung DK, et al. A novel semisynthesized small molecule icaritin reduces incidence of steroid-associated osteonecrosis with inhibition of both thrombosis and lipid-deposition in a dose-dependent manner. Bone. 2009; 44: 345-56.

37. Li J, Ge Z, Fan L, Wang K. Protective effects of molecular hydrogen on steroid-induced osteonecrosis in rabbits via reducing oxidative stress and apoptosis. BMC Musculoskelet Disord. 2017; 18: 58

38. Kanzaki H, Shinohara F, Kanako I, Yamaguchi Y, Fukaya S, Miyamoto Y, et al. Molecular regulatory mechanisms of osteoclastogenesis through cytoprotective enzymes. Redox Biol. 2016; 8: 186-91.

39. Ichiseki T, Kaneuji A, Katsuda S, Ueda Y, Sugimori T, Matsumoto T. DNA oxidation injury in bone early after steroid administration is involved in the pathogenesis of steroid-induced osteonecrosis. Rheumatology (Oxford). 2005; 44: 456-60.

40. Tsuchiya M, Ichiseki T, Ueda S, Ueda Y, Shimazaki M, Kaneuji A, et al. Mitochondrial stress and redox failure in steroid-associated osteonecrosis. Int J Med Sci. 2018; 15: 205-9.

41. Kim TH, Hong JM, Oh B, Cho YS, Lee JY, Kim HL, et al. Genetic association study of polymorphisms in the catalase gene with the risk of osteonecrosis of the femoral head in the Korean population. Osteoarthritis Cartilage. 2008; 16: 1060-6.

42. Glorieux C, Calderon PB. Catalase, a remarkable enzyme: targeting the oldest antioxidant enzyme to find a new cancer treatment approach. Biol Chem. 2017; 398: 1095-108.

43. Ichiseki T, Matsumoto T, Nishino M, Kaneuji A, Katsuda S. Oxidative stress and vascular permeability in steroid-induced osteonecrosis model. J Orthop Sci. 2004; 9: 509-15.

44. Liu Y, Wang C, Wang G, Sun Y, Deng Z, Chen L, et al. Loureirin B suppresses RANKL-induced osteoclastogenesis and ovariectomized osteoporosis via attenuating NFATc1 and ROS activities. Theranostics. 2019; 9: 4648-62.

45. Jung Y, Kim H, Min SH, Rhee SG, Jeong W. Dynein light chain LC8 negatively regulates NF-kappaB through the redox-dependent interaction with IkappaBalpha. J Biol Chem. 2008; 283: 23863-71.

46. Zheng LZ, Wang JL, Kong L, Huang L, Tian L, Pang QQ, et al. Steroid-associated osteonecrosis animal model in rats. J Orthop Translat. 2018; 13: $13-24$.

47. Han L, Wang B, Wang R, Gong S, Chen G, Xu W. The shift in the balance between osteoblastogenesis and adipogenesis of mesenchymal stem cells mediated by glucocorticoid receptor. Stem Cell Res Ther. 2019; 10: 377.

48. $\mathrm{Lu} \mathrm{BB}, \mathrm{Li} \mathrm{KH}$. Lipoic acid prevents steroid-induced osteonecrosis in rabbits. Rheumatol Int. 2012; 32: 1679-83.

49. Nozaki Y, Kumagai K, Miyata N, Niwa M. Pravastatin reduces steroid-induced osteonecrosis of the femoral head in SHRSP rats. Acta Orthop. 2012; 83: 87-92.

50. Sun ZB, Wang JW, Xiao H, Zhang QS, Kan WS, Mo FB, et al. Icariin may benefit the mesenchymal stem cells of patients with steroid-associated osteonecrosis by ABCB1-promoter demethylation: a preliminary study. Osteoporos Int. 2015; 26: 187-97.

51. Shidara K, Mohan G, Evan Lay YA, Jepsen KJ, Yao W, Lane NE. Strain-specific differences in the development of bone loss and incidence of osteonecrosis following glucocorticoid treatment in two different mouse strains. J Orthop Translat. 2019; 16: 91-101. 